$01 ; 14$

\title{
Модифицированный метод флуктуационного анализа нестационарных процессов
}

\author{
() А.Н. Павлов ${ }^{1,2}$, О.Н. Павлова ${ }^{1}$, А.А. Короновский (мл. $)^{1}$ \\ ${ }^{1}$ Саратовский национальный исследовательский государственный университет им. Н.Г. Чернышевского, Саратов, Россия \\ ${ }^{2}$ Саратовский государственный технический университет им. Гагарина Ю.А., Саратов, Россия \\ E-mail: pavlov.alexeyn@gmail.com
}

Поступило в Редакцию 29 ноября 2019г.

В окончательной редакции 29 ноября 2019 г.

Принято к публикации 20 декабря 2019г.

\begin{abstract}
Рассматривается метод анализа флуктуаций относительно тренда (метод DFA), позволяющий изучать длительные корреляции в нестационарных процессах. Предлагается его модификация, предусматривающая расчет дополнительной величины - показателя скейлинга, характеризующего эффекты нестационарности в экспериментальных данных. На примере динамики скорости кровотока в церебральных сосудах продемонстрированы возможности количественного описания изменений структуры сигналов с использованием предложенной модификации метода DFA.
\end{abstract}

Ключевые слова: нестационарный процесс, корреляционный анализ, флуктуации, скейлинг.

DOI: 10.21883/PJTF.2020.06.49166.18136

Нестационарная динамика систем с меняющимися во времени характеристиками ограничивает применимость классических методов спектрально-корреляционного анализа. По этой причине было предложено несколько альтернативных подходов к исследованию длительных корреляций в экспериментальных данных, среди которых чаще других используется метод анализа флуктуаций относительно тренда (detrended fluctuation analysis, DFA) [1,2]. Метод DFA имеет две характерные особенности: 1) вместо спадающей корреляционной функции он вводит в рассмотрение возрастающую функцию, которая обеспечивает более надежное оценивание степенных закономерностей для длительных корреляций, особенно при наличии помех и ограничений объема выборки; 2) составной частью алгоритма вычислений являются аппроксимация и последующее устранение низкочастотного тренда, позволяющие применять метод как для стационарных, так и для нестационарных процессов без их предварительной фильтрации. Эти обстоятельства определили широкое использование DFA, например, при обработке экспериментальных данных в физиологии и медицине [3-5], а также в различных областях физики [6-12]. Несмотря на значительное число опубликованных работ, возможность изучения сильно нестационарных данных на основе DFA продолжает дискутироваться. Некоторые исследователи полагают, что в этом случае сказываются ограничения метода и необходимо применять подходы, не использующие процедуру аппроксимации тренда [13], тогда как другие коллективы продолжают отдавать предпочтение именно DFA [14-16].

В настоящей работе мы предлагаем модификацию метода анализа флуктуаций относительно тренда для сильно нестационарных процессов, которая предусматривает расчет дополнительной характеристики - показателя скейлинга, описывающего эффекты нестационарности. На примере динамики церебрального кровотока мы проиллюстрируем возможности количественного описания изменений структуры сигналов с использованием предлагаемой модификации.

В первоначальном варианте метод DFA включал следующие операции: построение профиля сигнала $x(i)$, $i=1, \ldots, N$,

$$
y(k)=\sum_{i=1}^{k}[x(i)-\langle x\rangle],\langle x\rangle=\sum_{i=1}^{N} x(i), k=1, \ldots, N,
$$

разделение профиля $y(k)$ на сегменты одинаковой длины $n$ с аппроксимацией локального тренда $y_{n}(k)$ в пределах каждого сегмента и расчет среднеквадратичного отклонения

$$
F(n)=\sqrt{\frac{1}{N} \sum_{k=1}^{N}\left[y(k)-y_{n}(k)\right]^{2}} \sim n^{\alpha}
$$

Аппроксимация обычно осуществляется с помощью кусочно-линейных функций или полиномов. Для вычисления величины $\alpha$ (показателя степенной зависимости (2) или показателя скейлинга метода DFA [1,2]) сегментация профиля и расчет $F(n)$ должны проводиться в широком диапазоне значений $n$. Показатель $\alpha$ характеризует наличие положительных корреляций $(\alpha>0.5)$ и антикорреляций $(\alpha<0.5)$. Для стационарных процессов он связан с показателями, описывающими спад автокорреляционной функции и спектральной плотности мощности [17], хотя оценки скорости спада могут различаться для разных подходов [18]. 

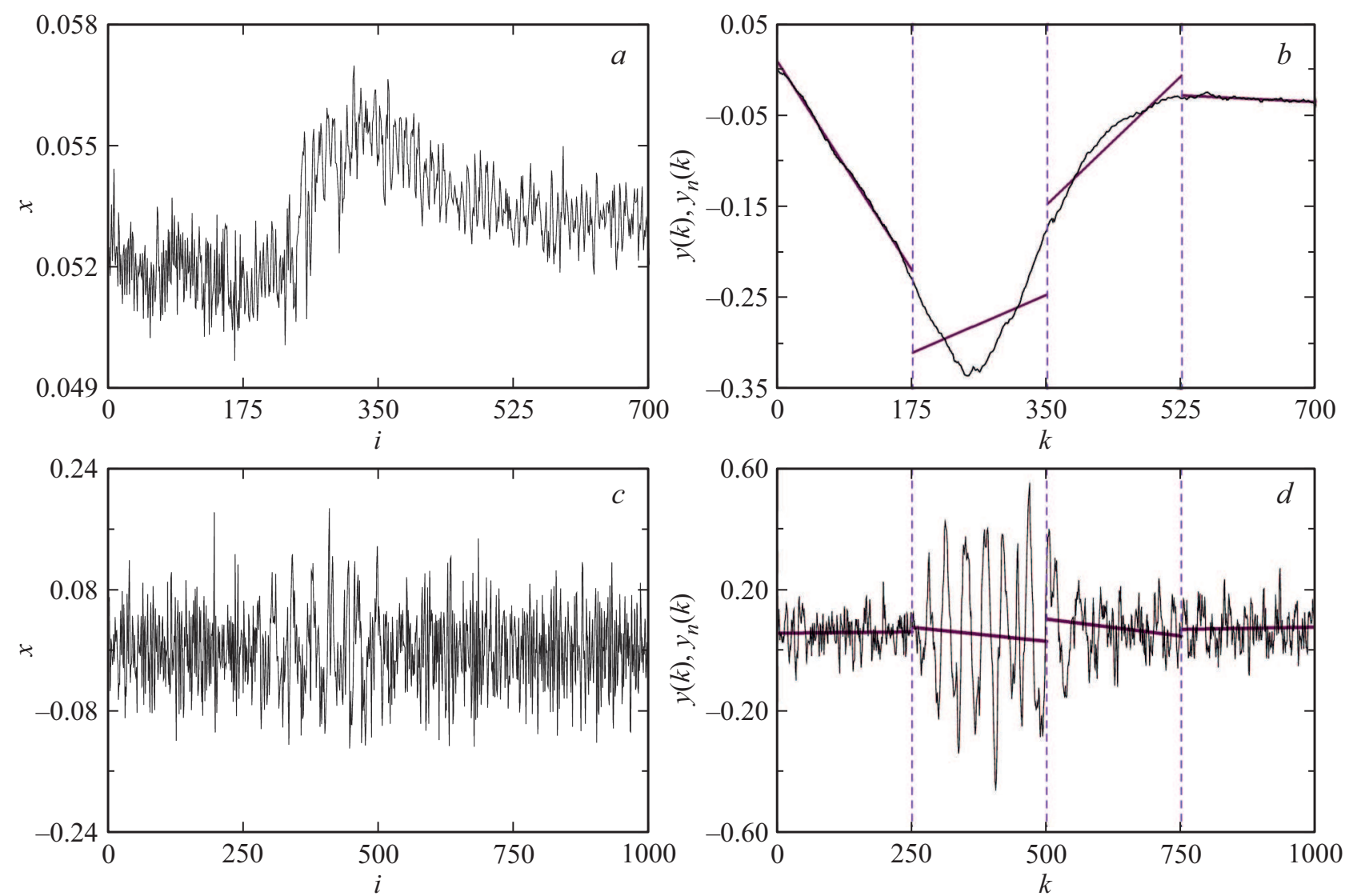

Рис. 1. Анализируемые нестационарные процессы, демонстрирующие изменения среднего уровня $(a)$ и чередование участков с разными статистическими характеристиками $(c)$, и соответствующие им профили с кусочно-линейной аппроксимацией тренда $(b, d)$. В обоих примерах среднеквадратичное отклонение профиля от линейной аппроксимации тренда значительно больше для второго сегмента по сравнению с остальными.

Рассмотрим нестационарный процесс, содержащий значительные изменения среднего уровня (рис. $1, a$ ) или чередование участков с разными статистическими характеристиками (рис. 1,c). В обоих примерах стандартные отклонения от аппроксимирующей функции сильно различаются для разных сегментов (рис. $1, b, d$ ), и если в первом случае низкочастотный тренд можно устранить на этапе предварительной обработки, проведя фильтрацию, то во втором случае фильтрация может не повлиять на нестационарность анализируемых данных, если она связана, например, с изменением дисперсии сигнала при постоянном среднем уровне. В рамках метода DFA отличия локальных значений стандартных отклонений, вычисленных для разных сегментов, не учитываются, хотя они могут оказывать существенное влияние на зависимость $F(n)$. Мы предлагаем ввести в рассмотрение дополнительную меру, которая характеризует эффекты нестационарности:

$$
d F(n)=\max \left[F_{l o c}(n)\right]-\min \left[F_{l o c}(n)\right],
$$

где $F_{l o c}(n)$ - локальные среднеквадратичные отклонения профиля сигнала $y(k)$ от аппроксимации тренда $y_{n}(k)$, вычисленные в пределах одного сегмента. Для стационарных процессов при заданном $n$ разброс значений $F_{l o c}(n)$ будет сравнительно небольшим, и величина $d F(n)$ приближается к нулю. При наличии сильной нестационарности $d F(n)$ принимает значения в диапазоне от нуля до $\max \left[F_{l o c}(n)\right]$. Обычно наблюдаются изменения $d F(n)$ при увеличении $n$, и соответствующая степенная зависимость описывается другим показателем скейлинга

$$
d F(n) \sim n^{\beta} .
$$

На рис. 2 приведен пример различий показателей $\alpha$ и $\beta$ для сигнала, изображенного на рис. $1, a$. Отметим, что в обоих случаях приведенные зависимости близки к линейным при выборе логарифмического масштаба по обеим осям, что свидетельствует о выполнении степенных закономерностей для $F(n)$ и $d F(n)$, которые описываются соответственно формулами (2) и (4).

Проиллюстрируем применение предлагаемого модифицированного подхода для решения задачи диагностики функциональных изменений в динамике церебральных кровеносных сосудов крыс при скачкообразном увеличении периферического артериального давления. В данной задаче важен анализ переходных процессов, позволяющих изучать резервные возможности организ- 


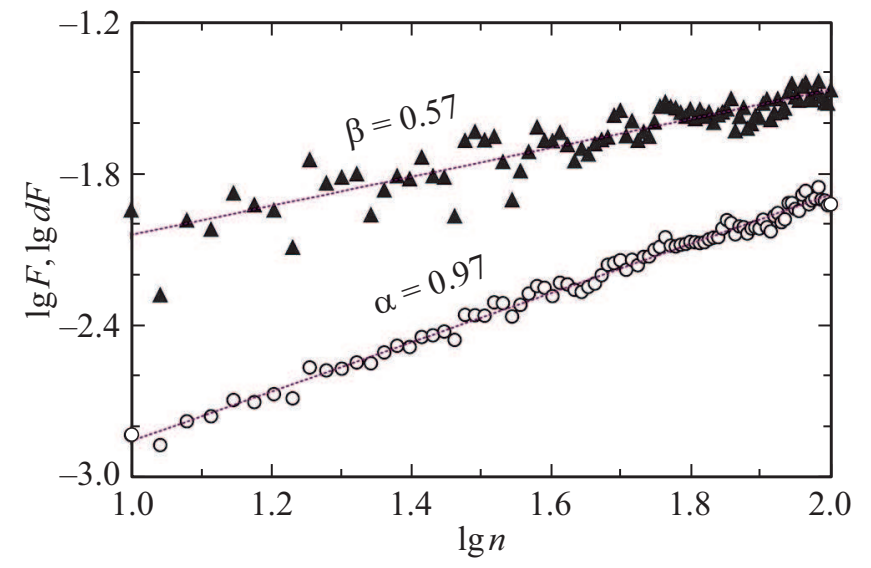

Рис. 2. Зависимости $F(n)$ и $d F(n)$ в логарифмическом масштабе для сигнала, изображенного на рис. $1, a$, показывающие различие показателей $\alpha$ и $\beta$.

ма. Описание методики проводимых физиологических экспериментов приведено в работе [19]. Регистрация сигналов церебрального кровотока в крупных и мелких кровеносных сосудах в течение $5 \mathrm{~min}$ осуществлялась методом лазерной спекл-интерферометрии [20] в соответствии с практическими рекомендациями [21]. Этот метод обеспечивает возможность исследования сравнительно больших участков без процедуры сканирования, например позволяет осуществлять одновременную запись относительной скорости кровотока в крупном сосуде и в сети окружающих его капилляров (проводя усреднение по выбранному фрагменту с использованием метода гистограмм [21]). Рассматривались две группы животных: в контрольном состоянии и при резком (приблизительно двукратном) повышении периферического артериального давления, вызванного введением мезатона. Наличие защитных механизмов препятствует соответствующей реакции кровеносных сосудов головного мозга, и относительная скорость церебрального кровотока сравнительно слабо меняется как в венах (увеличение составляет в среднем 2-3\%), так и в сети капилляров (9-11\%). Расчет показателя скейлинга $\alpha$, характеризующего длительные корреляции, также продемонстрировал относительно слабые реакции (около 6\% для макроциркуляции и 3\% для микроциркуляции), которые сопоставимы со статистическими погрешностями при анализе небольшой выборки (десять лабораторных животных). Вычисление показателя скейлинга $\beta$ позволило диагностировать более выраженные изменения, которые примерно в 2 раза различаются для вен и капилляров по сравнению с контрольным состоянием (21\% для макроциркуляции и $45 \%$ для микроциркуляции). Отметим при этом, что из рассмотрения исключался начальный участок сигнала непосредственно после скачка периферического артериального давления (2 min), который ассоциируется с наиболее заметными вариациями локального среднего уровня. Полученные результаты свидетельствуют о том, что привлечение дополнительной характеристики в рамках предложенного модифицированного метода DFA позволяет расширить возможности диагностики структурных изменений динамики при изменении условий функционирования организма.

В настоящей работе динамика церебральных кровеносных сосудов выбрана в качестве иллюстративного примера, показывающего преимущества модификации метода анализа флуктуаций относительно тренда. Однако возможности предложенного подхода значительно шире, и он может применяться для исследования структуры нестационарных процессов в различных областях науки и техники.

\section{Финансирование работы}

Исследование выполнено при поддержке гранта Российского научного фонда (проект № 19-12-00037).

\section{Соблюдение этических стандартов}

Все применимые международные, национальные и/или институциональные принципы ухода и использования животных были соблюдены.

\section{Конфликт интересов}

Авторы заявляют, что у них нет конфликта интересов.

\section{Список литературы}

[1] Peng C.-K., Buldyrev S.V., Havlin S., Simons M., Stanley H.E., Goldberger A.L. // Phys. Rev. E. 1994. V. 49. P. $1685-1689$.

[2] Peng C.-K., Havlin S., Stanley H.E., Goldberger A.L. // Chaos. 1995. V. 5. P. 82-87.

[3] Kuznetsov N.A, Rhea C.K. // PLoS ONE. 2017. V. 12. P. e0174144.

[4] Frolov N.S., Grubov V.V., Maksimenko V.A., Lüttjohann A., Makarov V.V., Pavlov A.N., Sitnikova E., Pisarchik A.N., Kurths J., Hramov A.E. // Sci. Rep. 2019. V. 9. P. 7243.

[5] Nolte G., Aburidi M., Engel A.K. // Sci. Rep. 2019. V. 9. P. 6339.

[6] Kiyono K., Tsujimoto Y. // Physica A. 2016. V. 462. P. $807-$ 815.

[7] Bhoumik G., Deb A., Bhattacharyya S., Ghosh D. // Adv. High Energy Phys. 2016. V. 2016. P. 7287803.

[8] Lovsletten O. // Phys. Rev. E. 2017. V. 96. P. 012141.

[9] Pavlova O.N., Abdurashitov A.S., Ulanova M.V., Shushunova N.A., Pavlov A.N. // Commun. Nonlinear Sci. Numer. Simul. 2019. V. 66. P. 31-40.

[10] Pavlova O.N., Pavlov A.N. // Physica A. 2019. V. 536. P. 122586.

[11] Павлов А.Н., Руннова А.Е., Максименко В.А., Павлова О.Н., Гришина Д.С., Храмов А.Е. // Письма в ЖТФ. 2019. T. 45. В. 4. C. $8-10$.

[12] Павлова О.Н., Павлов А.Н. // Письма в ЖТФ. 2019. Т. 45. B. 18. C. 6-9. 
[13] Bryce R.M., Sprague K.B. // Sci. Rep. 2012. V. 2. P. 315.

[14] Hu K., Ivanov P.C., Chen Z., Carpena P., Stanley H.E. // Phys. Rev. E. 2001. V. 64. P. 011114.

[15] Chen Z., Ivanov P.C., Hu K., Stanley H.E. // Phys. Rev. E. 2002. V. 65. P. 041107.

[16] Shao Y.H., Gu G.F., Jiang Z.Q., Zhou W.X., Sornette D. // Sci. Rep. 2012. V. 2. P. 835.

[17] Höll M., Kantz H. // Eur. Phys. J. B. 2015. V. 88. P. 1-7.

[18] Павлов А.Н., Павлова О.Н. // Письма в ЖТФ. 2008. Т. 34. B. 7. C. 71-78.

[19] Semyachkina-Glushkovskaya O.V., Abdurashitov A.S., Sindeev S.S., Tuchin V.V. // Quant. Electron. 2016. V. 46. P. 496-501.

[20] Boas D.A., Dunn A.K. // J. Biomed. Opt. 2010. V. 15. P. 011109.

[21] Abdurashitov A.S., Lychagov V.V., Sindeeva O.A., Semyachkina-Glushkovskaya O.V., Tuchin V.V. I/ Front. Optoelectron. 2015. V. 8. P. 187-194. 